\title{
Electrostatic Zach Phase Plates for Transmission Electron Microscopy: Status and Future Investigations
}

\author{
Martin Obermair ${ }^{1}$, Simon Hettler ${ }^{1}$, Manuel Dries ${ }^{1}$, Dagmar Gerthsen ${ }^{1}$ \\ 1. Laboratory for Electron Microscopy, Karlsruhe Institute of Technology, Karlsruhe, Germany.
}

The weak contrast of life-science objects in transmission electron microscopy (TEM) has led to a strong interest in the development of physical phase plates (PPs). By inducing a relative phase shift between scattered and unscattered electrons in the back focal plane (BFP) of the objective lens, PPs allow the generation of phase contrast without the need of applying a strong defocus. A large number of PP concepts exist [1], which can be mainly divided into thin-film and electrostatic approaches. Thin-film PPs exploit the mean inner potential of a structured film [2] or use the formation of a charge on a uniform film under illumination with the intense zero-order beam $[3,4]$ to generate the required relative phase shift.

However, the application of thin-film PPs is limited due to the fixed phase shift value and the scattering of electrons in the PP material. These limitations do not exist for electrostatic PPs, where a strongly localized electrostatic potential induces an adjustable phase shift on the unscattered electrons. Here we present our latest results achieved with the electrostatic Zach PP, which consists of a single rod carrying an isolated and shielded open gold electrode (Figure 1). When a voltage is applied to the electrode, an inhomogeneous electrostatic potential is formed at the tip. For the presented experiments, the Zach PP is implemented in the BFP of a Philips CM200 FEG/ST. The tip is positioned close to the zero-order beam, so that the unscattered electrons propagate through the electrostatic potential and acquire a phase shift which depends on the applied voltage.

Figure 2 shows the application of the Zach PP to a carbon nanotube (CNT) sample. Bundles of CNTs are dispersed on a holey amorphous carbon $(\mathrm{aC})$ film, so that free-standing CNTs can be investigated in vacuum regions. The contrast of the CNT bundles can be changed by applying different voltages and is also dependent on their orientation with respect to the PP rod. CNTs located perpendicular to the PP rod (yellow arrows) show a contrast inversion when the PP voltage is changed from $-5 \mathrm{~V}$ to $5 \mathrm{~V}$. CNTs located parallel to the PP rod (green arrows) show a contrast enhancement at $-5 \mathrm{~V}$, but disappear if a voltage of $5 \mathrm{~V}$ is applied to the electrode. The observed contrast results from the interplay of the inhomogeneous electrostatic potential, the obstruction of electrons by the PP rod and the astigmatism visible in the corresponding power spectra.

An application of Zach PPs is not only promising for biological weak-phase objects but is also beneficial in high-resolution TEM [5]. Figure 3 shows the application of a Zach PP to a germanium (Ge) sample in [101] zone axis orientation. The image resolution is not affected by the PP structure (Figure 3a) and the induced phase shift is visualized by a shift of the Thon rings in the power spectra (Figure $3 \mathrm{~b}$ ). Future investigations comprise the reconstruction of the object wave of crystalline samples using a Zach PP [6].

\section{References:}

[1] R M Glaeser, Rev. Sci. Instrum. 84 (2013), 111101.

[2] R Danev, K Nagayama, Ultramicroscopy 88 (2001), 243-252. 
[3] M Malac et al, Ultramicroscopy 118 (2012), 77-89.

[4] R Danev et al, PNAS 111 (2014), 15635-15640.

[5] S Hettler et al, New J Phys 18 (2016), 053005.

[6] The authors acknowledge funding from the Deutsche Forschungsgemeinschaft (DFG).
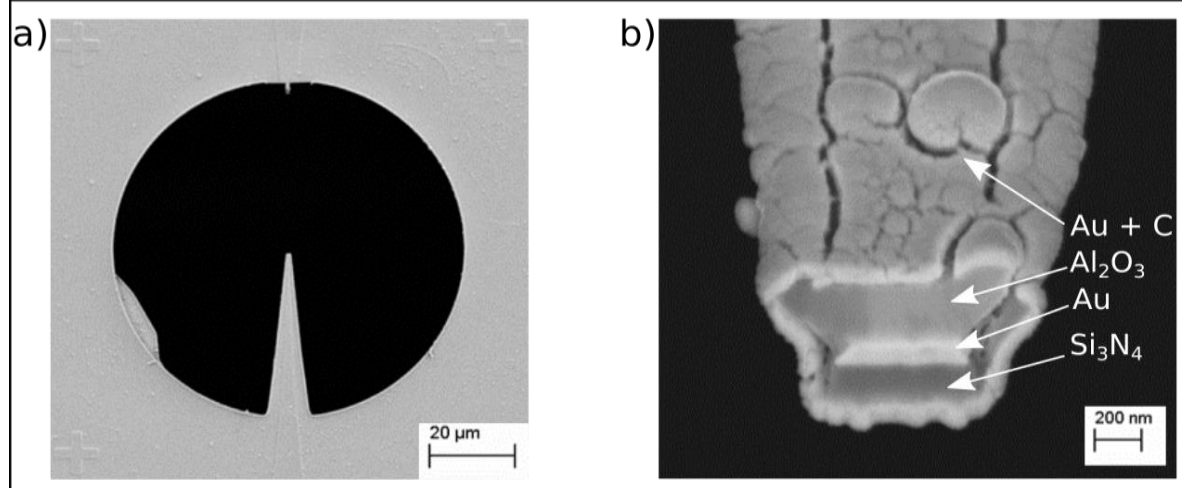

Figure 1. Scanning electron microscopy images of the used Zach PP. (a) Overview image shows the round aperture with the single rod. (b) The layer system of the Zach PP is visible in the image of the open tip of the PP rod.

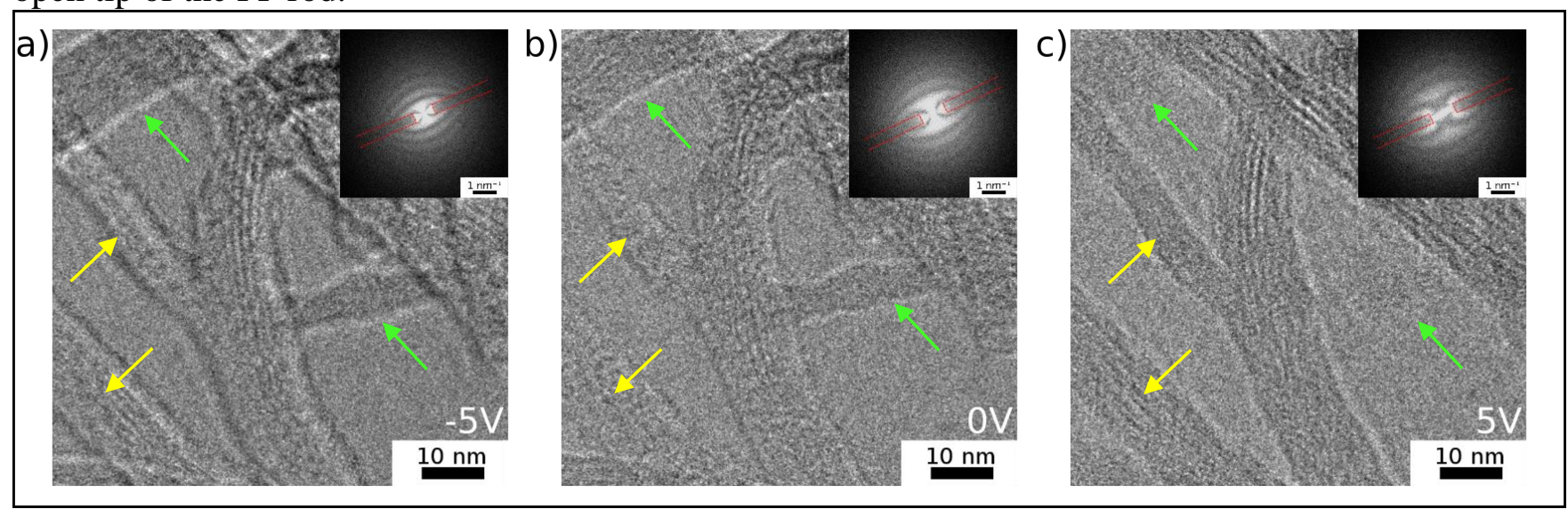

Figure 2. Zach PP TEM images of a CNT sample. (a-c) Images of a voltage series with $-5 \mathrm{~V}, 0 \mathrm{~V}$ and 5 $\mathrm{V}$ applied to the Zach PP electrode show an orientation-dependent contrast change of the CNTs. The position of the Zach PP rod is marked in red in the corresponding power spectra.

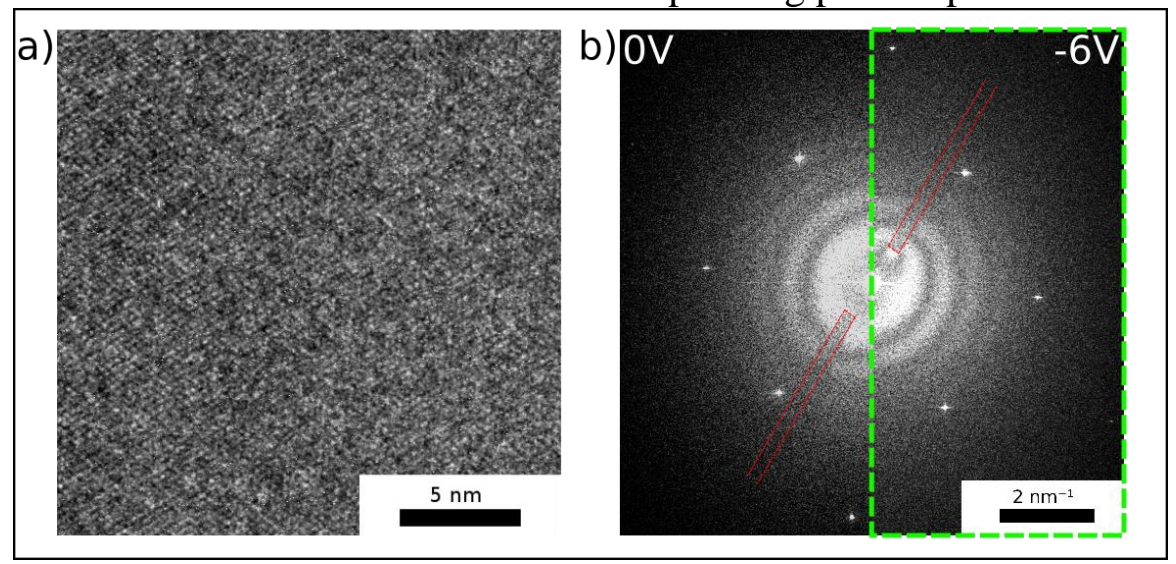

Figure 3. (a) High-resolution Zach PP TEM image of a Ge sample in [110] zone-axis orientation (b) The comparison between power spectra of two high-resolution TEM images with $0 \mathrm{~V}$ and $-6 \mathrm{~V}$ applied to the Zach PP electrode visualize the phase shift by the shift of Thon rings. 